\title{
Polatuzumab Vedotin: First Global Approval
}

\author{
Emma D. Deeks ${ }^{1}$ \\ Published online: 27 July 2019 \\ (c) Springer Nature 2019, corrected publication 2019
}

\begin{abstract}
Polatuzumab vedotin (polatuzumab vedotin-piiq; Polivy ${ }^{\mathrm{TM}}$ ) is an antibody-drug conjugate comprising a monoclonal antibody against CD79b (a B cell receptor component) covalently conjugated to the anti-mitotic cytotoxic agent monomethyl auristatin (MMAE) via a cleavable linker. After binding to CD79b on the B-cell surface, polatuzumab vedotin is internalized and the linker is cleaved, releasing MMAE into the cell, where it inhibits division and induces apoptosis. Polatuzumab vedotin is being developed by Genentech (a subsidiary of Roche) for the treatment of haematological malignancies. In June 2019, the US FDA granted accelerated approval to polatuzumab vedotin, in combination with bendamustine plus rituximab, for the treatment of adults with relapsed/refractory diffuse large B-cell lymphoma (DLBCL) who have received at least two prior therapies. Use of the compound in combination with bendamustine plus rituximab is also under regulatory review for relapsed/refractory DLBCL in the EU and is in ongoing phase 1b/2 development in this setting or relapsed/refractory follicular lymphoma (FL) in several countries. Various other polatuzumab vedotin combination therapy regimens are also in phase 1b/2 development for relapsed/refractory non-Hodgkin lymphoma (NHL) [including DLBCL and FL] or in phase 2 or 3 development for previously untreated DLBCL, while polatuzumab vedotin monotherapy has been in phase 1 development for relapsed/refractory B-cell NHL in Japan. This article summarizes the milestones in the development of polatuzumab vedotin leading to this first approval for its use in combination with bendamustine plus rituximab for relapsed/refractory DLBCL.
\end{abstract}

\section{Polatuzumab vedotin (Polivy $\left.{ }^{\mathrm{Tm}}\right)$ : key points}

An antibody-drug conjugate being developed by Genentech for the treatment of haematological malignancies

Received its first global approval on June 10th 2019 in the USA

Approved for use in combination with bendamustine plus rituximab as a treatment for adults with relapsed/ refractory DLBCL, not otherwise specified, after at least two prior therapies

Additional information for this Adis Insight Report can be found at https://doi.org/10.6084/m9.figshare.8980283.

This profile has been extracted and modified from the AdisInsight database. AdisInsight tracks drug development worldwide through the entire development process, from discovery, through preclinical and clinical studies to market launch and beyond.

Emma D. Deeks

dru@adis.com

1 Springer Nature, Private Bag 65901, Mairangi Bay, 0754 Auckland, New Zealand

\section{Introduction}

Antibody-drug conjugates (ADCs) are relatively recent biopharmaceutical compounds combining the targeting ability of a monoclonal antibody with the cell-killing activity of a small-molecule cytotoxic agent too potent (and thus toxic) to be used in standard chemotherapy regimens [1]. The monoclonal antibody is specific to a particular tumourassociated antigen and is linked to the cytotoxic drug via a biodegradable chemical linker. Once the antibody has bound the antigen at the tumour cell surface, the ADC is internalized and the linker degraded, releasing the active cytotoxic agent within the cell, which then causes cell death [1].

Polatuzumab vedotin (polatuzumab vedotin-piiq; Polivy ${ }^{\mathrm{TM}}$ ) is an ADC comprising a monoclonal antibody against CD79b (a B-cell receptor component moderately to strongly expressed in a majority of malignant lymphomas [2]) covalently conjugated via a cleavable linker to the microtubule-disrupting anti-mitotic agent monomethyl auristatin (MMAE). Once polatuzumab vedotin is internalized and its linker cleaved, the released MMAE inhibits cell division and induces apoptosis [3]. Polatuzumab vedotin is being developed by Genentech (a subsidiary of Roche) for the treatment of haematological malignancies. 


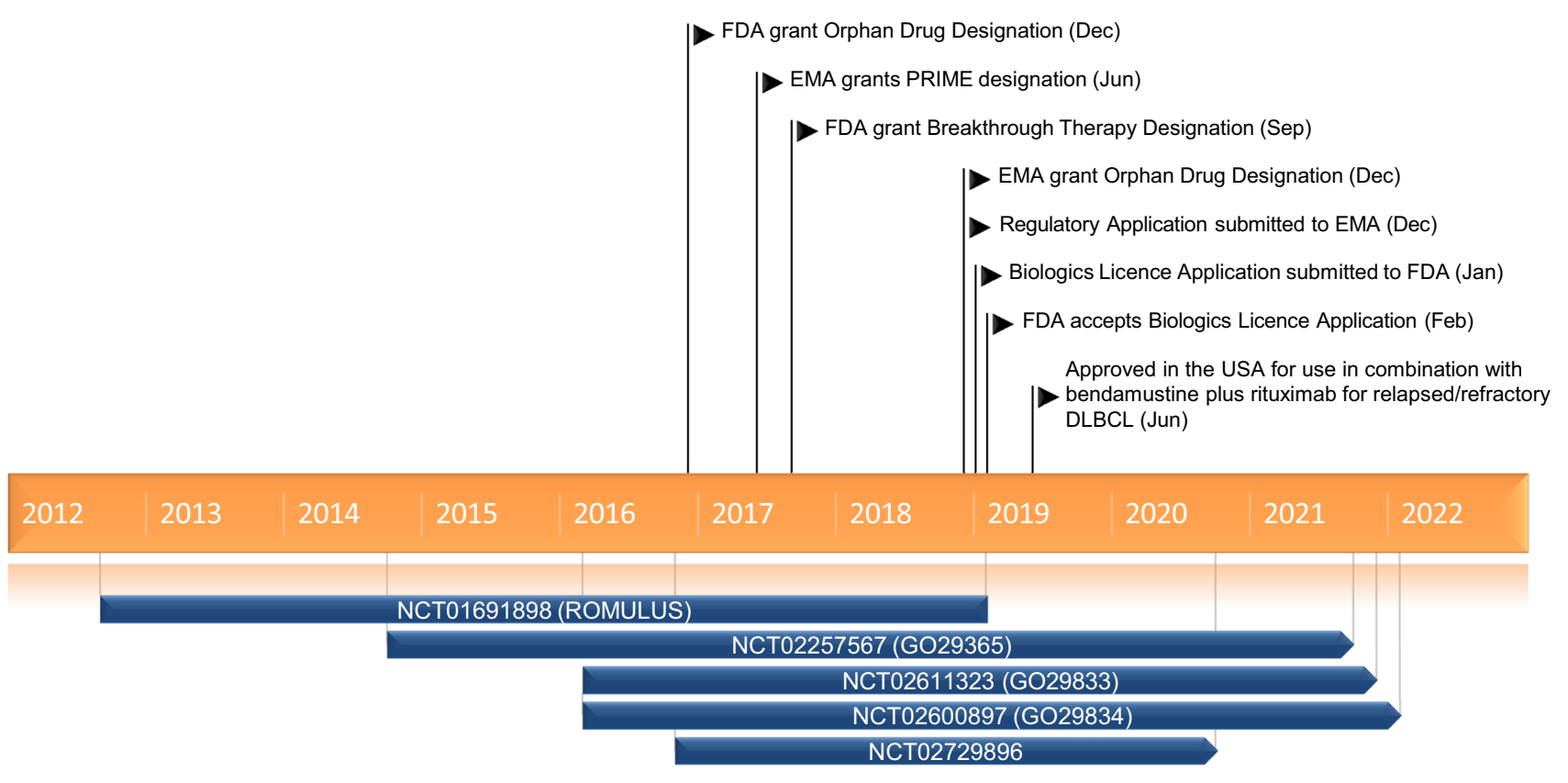

Key milestones in the development of polatuzumab vedotin in relapsed/refractory DLBCL, focussing on phase $1 \mathrm{~b} / 2$ trials. $D L B C L$ diffuse large B-cell lymphoma, PRIME PRIority MEdicines

In June 2019, the US FDA granted accelerated approval to polatuzumab vedotin (administered via intravenous infusion at a dosage of $1.8 \mathrm{mg} / \mathrm{kg}$ every 21 days for 6 cycles), in combination with bendamustine plus rituximab, for the treatment of adults with relapsed/refractory diffuse large B-cell lymphoma (DLBCL) who have received at least two prior therapies [4]. Use of polatuzumab vedotin in combination with bendamustine plus rituximab is also under regulatory review for relapsed/refractory DLBCL in the EU and is in ongoing phase $1 \mathrm{~b} / 2$ development in this setting or relapsed/refractory follicular lymphoma (FL) in several countries. Various other polatuzumab vedotin combination therapy regimens are also in phase $1 b / 2$ development for relapsed/refractory non-Hodgkin lymphoma (NHL) [including DLBCL and FL] or in phase 2 or 3 development for previously untreated DLBCL. Polatuzumab vedotin monotherapy has been in phase 1 development for relapsed/refractory B-cell NHL in Japan and was also assessed in relapsed/ refractory NHL or chronic lymphocytic leukaemia (CLL) in a first-in-human phase 1 trial in the USA, the Netherlands, France and Canada, although no development is planned for CLL. This article summarizes the milestones in the development of polatuzumab vedotin leading to this first approval for its use in combination with bendamustine plus rituximab for relapsed/refractory DLBCL.

\subsection{Company Agreements}

In April 2002, Seattle Genetics and Genentech entered into a multi-year ADC collaboration agreement, in which Seattle Genetics provide ADC technology and Genentech research, develop, manufacture, commercialize and pay royalties on the net sales of any resulting products. Genentech paid an upfront fee and was also to make an equity investment in Seattle Genetics and pay technology access/research fees and progress-dependent milestone payments. Upon achieving a specific benchmark, there is an option for Seattle Genetics to sell further equity to Genentech [5].

In July 2004, Seattle Genetics entered into a preferred provider agreement with Albany Molecular Research to manufacture its proprietary drug-linker system, an arrangement that also allowed ADC licensees of Seattle Genetics to work directly with Albany Molecular Research to obtain current good manufacturing practice supplies of drug-linker units for clinical trials [6].

Genentech expanded its collaboration with Seattle Genetics by designating additional antibody targets in December 2003 (for which Genentech paid \$US3.0 million and purchased \$US7.0 million of Seattle Genetics common stock to secure exclusive access) [7] and in November 2004 (for which Genentech agreed to pay a technology access fee of \$US1.6 million) [8]. Genentech paid \$US4.5 million in March 2007 [9] and \$9.5 million in April 2010 [10], for exclusive licences to specific targets and extension of the research term. Seattle Genetics and 
Genentech further expanded their agreement in August 2010, in which Genentech will pay \$US12 million for rights to use the ADC technology with additional antigens. Seattle Genetics may be eligible to receive over $\$$ US900 million in fees and milestone payments if all ADCs in the expanded collaboration are commercialized, as well as receiving royalties on sales [11].

\section{Scientific Summary}

\subsection{Pharmacodynamics}

Polatuzumab vedotin displayed activity against most DLBCL cell lines evaluated in vitro, regardless of whether they were of the activated B-cell-like (ABC) or germinal centre-like (GCL) cell-of-origin subtype or harboured mutations in $C D 79 B$ known to be associated with poor survival in DLBCL [12]. Almost all of the cell lines expressed BCL-XL and MYC, while few expressed BCL2 and MCL1; expression of these proteins can adversely impact survival and/or responses to chemotherapy in patients with DLBCL, although did not correlate with polatuzumab vedotin sensitivity [12].

Polatuzumab vedotin enhanced apoptosis and reduced proliferation of mature CD79b+ B-cell NHL cell lines (Burkitt lymphoma and primary mediastinal large B-cell lymphoma) [13] and increased overall survival (OS) in corresponding mouse xenograft models [14]. According to additional preclinical data, using polatuzumab vedotin in combination with other anti-cancer drugs, such as obinutuzumab or venetoclax, may result in an enhanced anti-neoplastic effect $[15,16]$.

Polatuzumab vedotin has potential for immunogenicity, as do other therapeutic proteins. Tests for anti-polatuzumab vedotin antibodies were positive in 8 of $134(6 \%)$ polatuzumab vedotin recipients across all arms of a pivotal phase $1 \mathrm{~b} / 2$ study (GO29365; Sect. 2.3.1.1) and in 14 of 536 (2.6\%) evaluable polatuzumab vedotin recipients across clinical trials [3], with the limited incidence of these antibodies precluding conclusions being made regarding their potential impact on the safety and efficacy of polatuzumab vedotin regimens [3].

Polatuzumab vedotin was not associated with clinically relevant QT interval prolongation in patients with B-cell haematological malignancies in clinical studies [3, 17]. For instance, in phase $1 / 2$ trials, polatuzumab vedotin $(1-2.4 \mathrm{mg} /$ $\mathrm{kg}$ every 21 days), alone or in combination with rituximab, was associated with mean increases from baseline in Fridericia's corrected QT interval of $<10 \mathrm{~ms}$, with no recipients experiencing changes of $>60 \mathrm{~ms}$ [17].

\subsection{Pharmacokinetics}

Both antibody-conjugated MMAE (acMMAE) and unconjugated MMAE display dose-proportional increases in plasma exposure across polatuzumab vedotin doses of $0.1-2.4 \mathrm{mg} /$ $\mathrm{kg}$ [3]. acMMAE exposure (as assessed by the area under the plasma concentration-time curve) at cycle 3 is predicted to be $\approx 30 \%$ higher than at cycle 1 and $>90 \%$ that at cycle 6. Plasma exposure to unconjugated MMAE is predicted to be $<3 \%$ that of acMMAE and to decrease with continued dosing every 21 days [3]. The estimated volume of distribution of acMMAE is $3.15 \mathrm{~L}$, according to population pharmacokinetic (popPK) analysis. MMAE is $71-77 \%$ bound to plasma proteins and has a blood : plasma ratio of 0.79-0.98 in vitro [3]. Polatuzumab vedotin is likely degraded into small peptides and amino acids, as well as unconjugated MMAE and catabolites of unconjugated MMAE. The terminal half-life of unconjugated MMAE is $\approx 4$ days after the initial dose of polatuzumab

Features and properties of polatuzumab vedotin

\begin{tabular}{|c|c|}
\hline Alternative names & $\begin{array}{l}\text { Polatuzumab vedotin-piiq; Polivy; anti-CD79b-VC-MMAE; DCDS-4501A; FCU-2711; RG-7596; RO-5541077; } \\
\text { RO-5541077-000 }\end{array}$ \\
\hline Class & Antineoplastics; auristatins; drug conjugates; immunotoxins; monoclonal antibodies \\
\hline Mechanism of Action & $\begin{array}{l}\text { After internalization by CD79b-expressing cells, cleavage of the monoclonal antibody-MMAE linker releases active } \\
\text { MMAE (an apoptosis stimulant and inhibitor of mitosis, tubulin and tubulin polymerization) }\end{array}$ \\
\hline Route of Administration & Intravenous infusion \\
\hline Pharmacodynamics & $\begin{array}{l}\text { Enhances apoptosis and reduces proliferation of mature } \mathrm{CD} 79 \mathrm{~b}+\mathrm{B} \text {-cell non-Hodgkin lymphoma cell lines and } \\
\text { increases overall survival in corresponding xenograft models; may have additional benefit in combination with } \\
\text { other anti-cancer agents }\end{array}$ \\
\hline Pharmacokinetics & Systemic exposure to unconjugated MMAE is predicted to be minimal relative to that of antibody-conjugated MMAE \\
\hline $\begin{array}{l}\text { Most frequent grade } \geq 3 \\
\text { adverse events }\end{array}$ & Cytopenias \\
\hline \multicolumn{2}{|l|}{ ATC codes } \\
\hline WHO ATC code & L01X-C (monoclonal antibodies) \\
\hline EphMRA ATC code & L1G (monoclonal antibody antineoplastics) \\
\hline Chemical Name & $\begin{array}{l}\text { Immunoglobulin G1-kappa auristatin E conjugate, anti-[Homo sapiens CD79B (immunoglobulin-associated CD79 } \\
\text { beta)], humanized monoclonal antibody conjugated to auristatin E }\end{array}$ \\
\hline
\end{tabular}

MMAE monomethyl auristatin 
vedotin, whereas that of acMMAE is $\approx 12$ days at cycle 6 ; the predicted clearance of acMMAE is $0.9 \mathrm{~L} /$ day [3].

Bodyweight was identified as a significant covariate for both the volume of distribution and clearance of acMMAE in a popPK analysis, with a simulation suggesting there may be less pharmacokinetic variability with bodyweight-based than with fixed dosing [18].

There appears to be no clinically relevant impact of patient age, sex or race/ethnicity (non-Asian vs. Asian [19]) on the pharmacokinetics of polatuzumab vedotin, of mild or moderate renal impairment on the pharmacokinetics of acMMAE or unconjugated MMAE, or of mild hepatic impairment on MMAE exposure [3]. Whether severe renal impairment (including end-stage renal disease, with or without dialysis), moderate to severe hepatic impairment or liver transplantation may impact acMMAE or unconjugated MMAE pharmacokinetics is not yet known. However, polatuzumab vedotin should be avoided in patients with moderate or severe hepatic impairment (i.e. bilirubin $>1.5$ times the upper limit of normal), as MMAE exposure is likely to be increased in these populations [3]. Greater acMMAE exposure and longer treatment duration may increase the likelihood of polatuzumab vedotin recipients experiencing peripheral neuropathy $[18,20]$, which is a common adverse event (AE) with the drug (Sect. 2.4).

\subsubsection{Potential Drug Interactions}

MMAE is a CYP3A4 substrate; thus, its exposure may increase or decrease if polatuzumab vedotin is coadministered with drugs that are inhibitors or inducers of CYP3A4 [3]. In vitro, MMAE is a substrate of p-glycoprotein, although it does not inhibit this transporter. MMAE is also not an inhibitor of CYP1A2, CYP2B6, CYP2C8, CYP2C9, CYP2C19 or CYP2D6 or an inducer of key CYP enzymes in vitro. Polatuzumab vedotin is predicted not to impact exposure to the CYP3A-sensitive substrate midazolam when the drugs are used concomitantly [3].

Polatuzumab vedotin had no clinically relevant interactions with regimens comprising either rituximab or obinutuzumab, with [21, 22] or without [22] bendamustine [22] or CHP (i.e. cyclophosphamide, doxorubicin and prednisone) $[21,22]$ in patients with NHL. Indeed, concomitant use of polatuzumab vedotin and bendamustine or rituximab was predicted to have no clinically relevant impact on the pharmacokinetics of acMMAE or unconjugated MMAE in popPK modelling [3].

\subsection{Therapeutic Trials}

\subsubsection{In Combination with Immunotherapy}

In a cohort of nine patients with relapsed/refractory B-cell NHL who received polatuzumab vedotin plus rituximab in an open-label phase 1 trial (NCT01290549), seven achieved a response, of which two were complete responses (CRs) and five were partial responses (PRs) [23]. The median duration of response (DoR) was 12.3 months and the median progression-free survival (PFS) was 12.5 months, with the latter outcome not clearly correlating with patient baseline characteristics. Patients had indolent B-cell lymphoma $(n=5)$, mantle-cell lymphoma (MCL) $[n=3]$ or transformed FL $(n=1)$ and received treatment in 21-day cycles, with polatuzumab vedotin administered intravenously at $2.4 \mathrm{mg} / \mathrm{kg}$ (on the basis of prior monotherapy dose-escalation cohorts; Sect. 2.3.2) [23].

Polatuzumab vedotin $2.4 \mathrm{mg} / \mathrm{kg}$ plus rituximab was also assessed in patients with relapsed/refractory DLBCL $(n=39)$ or FL $(n=20)$ in the phase 2 part of the ROMULUS phase 1b/2 trial (NCT01691898) [24]. In these respective patient populations, the objective response (OR) rate was $54 \%$ and $70 \%$, the CR rate $21 \%$ and $45 \%$, the PR rate $33 \%$ and $25 \%$ and the median DoR 13.4 and 9.4 months. The DLBCL and FL groups had a median PFS of 5.6 and 15.3 months, and the median OS was reached only in the DLBCL group (20.1 months). Clinical benefit was also evident in DLBCL and FL patients who were refractory to their last therapy ( $n=31$ and 7) [e.g. the OR rate was $45 \%$ and 57\%, the median DoR 13.4 and 9.4 months, the median PFS 4.7 and 13.6 months and the median OS 11.7 months or not yet reached] [24].

In this randomized, open-label trial, the efficacy of this polatuzumab vedotin plus rituximab regimen was generally not markedly different from that of the anti-CD22 ADC pinatuzumab vedotin likewise used at $2.4 \mathrm{mg} / \mathrm{kg}$ in combination with rituximab (42 DLBCL; 21 FL) [24]. However, there was a notable numerical difference between the polatuzumab vedotin and pinatuzumab vedotin regimens in terms of the CR rate in FL patients (45\% vs. 5\%) and the median DoR in DLBCL patients (13.4 vs. 6.2 months). Each regimen displayed antitumour efficacy across DLBCL cell-of-origin subtypes (ABC and GLC) and in patients with DLBCL expressing high levels of the anti-apoptotic regulator BCL2; tumour shrinkage did not correlate with expression levels of BCL2 or target proteins (i.e. CD79b for polatuzumab vedotin; CD22 for pinatuzumab vedotin). Polatuzumab vedotin or pinatuzumab vedotin was administered with rituximab, every 21 days until progressive disease or unacceptable toxicity, to a maximum of 1 year; all agents were administered intravenously and responses were investigator assessed (in accordance with revised International Working Group criteria for malignant lymphoma). Patients had received up to four prior systemic therapies, with $78 \%$ of those with DLBCL and $44 \%$ of those with FL being refractory to their last treatment [24].

Polatuzumab vedotin was also evaluated in combination with obinutuzumab in patients with relapsed/refractory 
DLBCL $(n=21)$ or FL $(n=23)$ in ROMULUS [25]. In the respective groups, the best OR (as assessed by PET and Lugano 2014 criteria) was CR in $29 \%$ and $30 \%$ of patients, PR in $24 \%$ and $48 \%$, stable disease in $5 \%$ and $22 \%$ and progressive disease in $43 \%$ and $0 \%$; the OR rate was $52 \%$ and $78 \%$. Patients received up to eight 21 -day cycles of intravenous polatuzumab vedotin $1.8 \mathrm{mg} / \mathrm{kg}$ in combination with obinutuzumab; the median number of treatment cycles was two in patients with DLBCL and five in those with FL. Participants had received $1-8$ prior therapies, with a majority (61\%) being refractory to the last [25].

2.3.1.1 Plus Chemotherapy Polatuzumab vedotin in combination with bendamustine and either rituximab or obinutuzumab has been evaluated as a treatment for relapsed/ refractory DLBCL or FL in an open-label phase $1 \mathrm{~b} /$ trial (NCT02257567; GO29365). These regimens showed clinical promise in the noncomparative phase $1 \mathrm{~b}$ safety runin $(n=12 \mathrm{DLBCL} ; n=12 \mathrm{FL})$ and/or phase 2 expansion $(n=21$ DLBCL; $n=20 \mathrm{FL})$ cohorts of the study [26, 27], with polatuzumab vedotin, bendamustine plus rituximab then also compared with bendamustine plus rituximab in randomized cohorts ( $n=80 \mathrm{DLBCL} ; n=80 \mathrm{FL})$. In the most recent analysis of the randomized data (cutoff April 2018) in patients with relapsed/refractory DLBCL, the combination of polatuzumab vedotin $1.8 \mathrm{mg} / \mathrm{kg}$, bendamustine plus rituximab $(n=40)$ was more favourable than bendamustine plus rituximab $(n=40)$ with regard to the rate of CR ( $40 \%$ vs. $18 \% ; p=0.026)$ and OR (45\% vs. $18 \% ; p$ value not reported) at the end of treatment [27, 28]. The DoR was also longer with this polatuzumab vedotin-containing regimen than the comparator regimen (10.3 vs. 4.1 months; $p=0.032$ ) [28] and, notably, among the 25 and 10 patients in the respective groups who achieved a best overall response of CR or PR, $64 \%$ and $30 \%$ had a DoR of $\geq 6$ months and $48 \%$ and $20 \%$ had a DoR of $\geq 12$ months [3]. The polatuzumab vedotin-containing regimen was associated with a longer median PFS (7.6 vs. 2.0 months; $p<0.0001$ ) and OS (12.4 vs. 4.7 months; $p=0.0023$ ) than the comparator regimen, with the benefit over the comparator regimen for these outcomes evident regardless of the cell-of-origin or MYC/ BCL2 double-expressor status [28]. Earlier analysis of the randomized cohort data supported these findings in patients with DLBCL (data cutoff May 2017), but found no marked benefit with polatuzumab vedotin, bendamustine plus rituximab $(n=39)$ versus bendamustine plus rituximab $(n=41)$ in patients with FL (data cutoff October 2017) in terms of CR (69\% vs. $63 \%$ ) or median PFS (17 vs. $17 \%$ ); the median OS had not yet been reached [29, 30]. Randomized patients received up to six 21-day cycles of treatment, with all drugs administered intravenously, including polatuzumab vedotin (day 2 of cycle 1; day 1 of cycles 2-6) [3]. Responses were assessed via independent review committee using modified
Lugano criteria (CR required PET negativity and bone marrow confirmation).

Other polatuzumab vedotin-based immunochemotherapy regimens have displayed promising clinical efficacy in patients with B-cell NHL in another open-label phase $1 \mathrm{~b} / 2$ trial (NCT01992653) [31]. In the phase 1b dose-escalation cohorts of the study, in which 25 patients with B-cell NHL (regardless of prior treatment status) received either polatuzumab vedotin $(1.0-1.8 \mathrm{mg} / \mathrm{kg})$ in combination with rituximab plus CHP, or polatuzumab vedotin ( 1.4 or $1.8 \mathrm{mg} / \mathrm{kg}$ ) in combination with obinutuzumab plus CHP, the OR rate at the end of treatment was high, regardless of whether the patients had DLBCL ( $n=9$ have data reported; all received polatuzumab vedotin $<1.8 \mathrm{mg} / \mathrm{kg}$ ) [OR rate $89 \%$; all but one was a CR] or other forms of NHL (including FL and MCL; $n=7$ ) [OR rate $100 \%$; all CRs]. The corresponding OR rate was likewise high (89\%; CR 77\% and PR 12\%) when polatuzumab vedotin $1.8 \mathrm{mg} / \mathrm{kg}$ was used in combination with these immunochemotherapy regimens in patients with previously untreated DLBCL in the dose-escalation $(n=9)$ or dose-expansion $(n=57)$ cohorts [31].

In these previously untreated patients $(n=66), 95 \%$ of responses were ongoing at 12 months, the 12- and 24-month PFS rates were $91 \%$ and $83 \%$, and the 12 -month OS rate was 94\%. Responses to treatment and PFS were not associated with CD79b expression (prespecified analyses) or markedly impacted by the DLBCL cell-of-origin. PFS was also not impacted by which immunotherapy agent was in the regimen, the number of planned treatment cycles, the International Prognostic Index (IPI) category or MYC/BCL2 double-expression (latter analysis prespecified; others were post hoc) [31]. At the time of this analysis (data cutoff December 2017), recipients of polatuzumab vedotin $1.8 \mathrm{mg} / \mathrm{kg}$ had a median study duration of 21.5 months. Earlier analyses were generally supportive of these findings [32-35]. Polatuzumab vedotin $1.8 \mathrm{mg} / \mathrm{kg}$ was recommended for the phase 2 doseexpansion cohorts on the basis of its safety and benefit-risk profile in the dose-escalation cohorts. Patients received six or eight 21-day cycles of treatment and responses were investigator assessed (based on an International Workshop to Standardize Response Criteria for NHL 2007, with CRs evaluated by PET).

2.3.1.2 Plus Immunomodulating Therapy A regimen comprising polatuzumab vedotin, obinutuzumab and the immunomodulating agent lenalidomide has shown promise as a treatment for relapsed/refractory FL in a phase $1 \mathrm{~b} / 2$ trial (NCT02600897; GO29834) [36]. At the pre-planned interim analysis ( $n=18$ evaluable for efficacy), the CR as per modified Lugano 2014 criteria at the end of induction treatment (a primary endpoint) was $61 \%$ when assessed by the investigator and $67 \%$ when assessed by independent review committee, with the best overall response rate being $89 \%$ regard- 
Key clinical trials of polatuzumab vedotin

\begin{tabular}{|c|c|c|c|c|c|c|}
\hline Drug(s) & Indication & Phase & Status & Location(s) & Identifier & Sponsor \\
\hline $\begin{array}{l}\text { Polatuzumab vedotin + bendamustine } \\
\quad \text { + rituximab vs. bendamustine } \\
\text { + rituximab; polatuzumab vedotin } \\
\text { + bendamustine + obinutuzumab }\end{array}$ & $\mathrm{r} / \mathrm{r}$ DLBCL or FL & $1 \mathrm{~b} / 2$ & Ongoing & Multinational & $\begin{array}{l}\text { NCT02257567 } \\
\quad(\text { GO29365) }\end{array}$ & $\begin{array}{l}\text { Hoffmann-La } \\
\text { Roche }\end{array}$ \\
\hline $\begin{array}{l}\text { Polatuzumab vedotin + bendamustine } \\
\text { +rituximab }\end{array}$ & $\mathrm{r} / \mathrm{r}$ DLBCL & 2 & Ongoing & Japan & JapicCTI184048 & $\begin{array}{l}\text { Chugai Phar- } \\
\text { maceutical }\end{array}$ \\
\hline $\begin{array}{l}\text { Polatuzumab vedotin + rituximab vs. } \\
\text { pinatuzumab vedotin + rituximab; } \\
\text { polatuzumab vedotin + obinutuzumab }\end{array}$ & $\mathrm{r} / \mathrm{r}$ DLBCL or FL & $1 b / 2$ & Completed & Multinational & $\begin{array}{l}\text { NCT01691898 } \\
\text { (ROMULUS) }\end{array}$ & Genentech Inc \\
\hline $\begin{array}{l}\text { Mosunetuzumab } \pm \text { polatuzumab } \\
\text { vedotin vs. polatuzumab }+ \\
\text { bendamustine }+ \text { rituximab }\end{array}$ & $\mathrm{r} / \mathrm{r}$ DLBCL & $1 \mathrm{~b} / 2$ & Recruiting & USA & NCT03671018 & $\begin{array}{l}\text { Hoffmann-La } \\
\text { Roche }\end{array}$ \\
\hline $\begin{array}{l}\text { Mosunetuzumab } \pm \text { polatuzumab vedotin } \\
\text { vs. investigator-chosen regimen }{ }^{\mathrm{a}}\end{array}$ & $\mathrm{r} / \mathrm{r} \mathrm{FL}$ & & & & & \\
\hline Polatuzumab vedotin & $\mathrm{r} / \mathrm{r}$ B-cell NHL & 1 & & Japan & $\begin{array}{l}\text { JapicCTI142580 } \\
\text { (JO29138) }\end{array}$ & $\begin{array}{l}\text { Chugai Phar- } \\
\text { maceutical }\end{array}$ \\
\hline Polatuzumab vedotin & $\mathrm{r} / \mathrm{r}$ NHL or CLL & 1 & Completed & USA, Canada, & NCT01290549 & Genentech Inc \\
\hline Polatuzumab vedotin + rituximab & $\mathrm{r} / \mathrm{r}$ NHL & & & $\begin{array}{l}\text { France, The } \\
\text { Netherlands }\end{array}$ & (DCS4968 g) & \\
\hline $\begin{array}{l}\text { Polatuzumab vedotin }+ \text { venetoclax }+ \\
\text { rituximab, then venetoclax }+ \text { rituximab }\end{array}$ & $\mathrm{r} / \mathrm{r}$ DLBCL & $1 \mathrm{~b} / 2$ & Recruiting & $\begin{array}{l}\text { USA, Australia, } \\
\text { Italy }\end{array}$ & $\begin{array}{l}\text { NCT02611323 } \\
\quad(\text { GO29833) }\end{array}$ & $\begin{array}{l}\text { Hoffmann-La } \\
\text { Roche }\end{array}$ \\
\hline $\begin{array}{l}\text { Polatuzumab vedotin }+ \text { venetoclax }+ \\
\text { obinutuzumab, then venetoclax }+ \\
\text { obinutuzumab }\end{array}$ & $\mathrm{r} / \mathrm{r} \mathrm{FL}$ & & & & & \\
\hline $\begin{array}{l}\text { Polatuzumab vedotin + lenalidomide } \\
\text { + rituximab, then lenalidomide } \\
\text { + rituximab }\end{array}$ & $\mathrm{r} / \mathrm{r}$ DLBCL & $1 \mathrm{~b} / 2$ & Recruiting & USA, Spain, UK & $\begin{array}{l}\text { NCT02600897 } \\
\quad(\text { GO29834) }\end{array}$ & $\begin{array}{l}\text { Hoffmann-La } \\
\text { Roche }\end{array}$ \\
\hline $\begin{array}{l}\text { Polatuzumab vedotin + lenalidomide } \\
\text { + obinutuzumab, then lenalidomide } \\
\text { + obinutuzumab }\end{array}$ & $\mathrm{r} / \mathrm{r} \mathrm{FL}$ & & & & & \\
\hline $\begin{array}{l}\text { Polatuzumab vedotin + atezolizumab } \\
+ \text { rituximab }\end{array}$ & $\mathrm{r} / \mathrm{r}$ DLBCL & $1 \mathrm{~b} / 2$ & Halted & $\begin{array}{l}\text { USA, Germany, } \\
\text { Poland }\end{array}$ & NCT02729896 & $\begin{array}{l}\text { Hoffmann-La } \\
\text { Roche }\end{array}$ \\
\hline $\begin{array}{l}\text { Polatuzumab vedotin + atezolizumab } \\
+ \text { obinutuzumab }\end{array}$ & $\mathrm{r} / \mathrm{r} \mathrm{FL}$ & & & & & \\
\hline $\begin{array}{l}\text { Polatuzumab vedotin + rituximab } \\
\text { + gemcitabine + oxaliplatin vs. } \\
\text { rituximab + gemcitabine + oxaliplatin }\end{array}$ & $\mathrm{r} / \mathrm{r}$ DLBCL & 3 & Planned & & Pending & Roche \\
\hline $\begin{array}{l}\text { Mosunetuzumab-CHOP or polatuzumab } \\
\text { vedotin }+ \text { mosunetuzumab-CHP }\end{array}$ & $\mathrm{r} / \mathrm{r}$ NHL & $1 \mathrm{~b}$ & Recruiting & $\begin{array}{l}\text { USA, Republic of } \\
\text { Korea }\end{array}$ & NCT03677141 & $\begin{array}{l}\text { Hoffmann-La } \\
\text { Roche }\end{array}$ \\
\hline $\begin{array}{l}\text { Mosunetuzumab-CHOP or polatuzumab } \\
\text { vedotin + mosunetuzumab-CHP vs. } \\
\text { polatuzumab vedotin + rituximab-CHP }\end{array}$ & $\mathrm{r} / \mathrm{r}$ DLBCL & 2 & & & & \\
\hline $\begin{array}{l}\text { Polatuzumab vedotin + rituximab-CHP } \\
\text { vs. rituximab-CHOP }\end{array}$ & $\begin{array}{l}\text { Previously } \\
\text { untreated DLBCL }\end{array}$ & 3 & Ongoing & Multinational & $\begin{array}{l}\text { NCT03274492 } \\
\text { (POLARIX) }\end{array}$ & $\begin{array}{l}\text { Hoffmann-La } \\
\text { Roche }\end{array}$ \\
\hline \multirow{2}{*}{$\begin{array}{l}\text { Polatuzumab vedotin + either rituximab- } \\
\text { CHP or obinutuzumab-CHP }\end{array}$} & NHL & $1 b$ & \multirow[t]{2}{*}{ Completed } & \multirow[t]{2}{*}{ USA, France } & \multirow{2}{*}{$\begin{array}{l}\text { NCT01992653 } \\
\quad(\text { GO29044) }\end{array}$} & \multirow[t]{2}{*}{ Genentech Inc } \\
\hline & $\begin{array}{l}\text { Previously } \\
\text { untreated DLBCL }\end{array}$ & 2 & & & & \\
\hline
\end{tabular}

CHOP cyclophosphamide, doxorubicin, vincristine + prednisone, $C H P$ cyclophosphamide, doxorubicin + prednisone, $C V P$ cyclophosphamide, vincristine + prednisone, $D L B C L$ diffuse large B-cell lymphoma, $F L$ follicular lymphoma, $N H L$ non-Hodgkin lymphoma, $r / r$ relapsed/refractory ${ }^{a}$ Rituximab-CHOP, rituximab-CVP or obinutuzumab + bendamustine, then obinutuzumab maintenance therapy

less of assessment method. The median PFS had not been reached after a median 8.95 months of follow-up. Induction consisted of six 28-day cycles of intravenous polatuzumab vedotin ( 1.4 or $1.8 \mathrm{mg} / \mathrm{kg}$, on day 1 of each cycle) plus intravenous obinutuzumab and oral lenalidomide, with data for patients who received polatuzumab vedotin $1.4 \mathrm{mg} / \mathrm{kg}$ being discussed here. Patients who achieved a CR, PR or stable disease after induction were eligible to receive maintenance treatment with obinutuzumab and lenalidomide. Most (79\%) of the 52 patients enrolled in this trial had received at least 
two prior lines of therapy and half were refractory to their last treatment [36].

\subsubsection{Monotherapy}

Polatuzumab vedotin monotherapy was evaluated in patients with relapsed/refractory B-cell NHL or CLL in a phase 1 study (NCT01290549) [23]. There were two dose-escalation cohorts: one in $34 \mathrm{NHL}$ patients $(0.1-2.4 \mathrm{mg} / \mathrm{kg})$ and the other in 18 CLL patients $(0.25-1.8 \mathrm{mg} / \mathrm{kg})$. These determined $2.4 \mathrm{mg} / \mathrm{kg}$ (based on pharmacokinetics, safety/ tolerability and dose-limiting toxicities, of which grade 4 neutropenia was the only one) as the dose most suitable for further evaluation in NHL in a dose-expansion cohort $(n=34)$ and phase 2 studies, and $1.0 \mathrm{mg} / \mathrm{kg}$ as the maximum tolerated dose for CLL; however, as no ORs occurred with polatuzumab vedotin in the latter setting, CLL enrollment was stopped. Among the 68 NHL patients who received polatuzumab vedotin in the escalation or expansion phases (which included 25 with indolent B-cell lymphoma, 39 with DLBCL and 4 with MCL), the highest rates of OR occurred with doses of $\geq 1.8 \mathrm{mg} / \mathrm{kg}$. There were 42 patients with NHL who received polatuzumab vedotin $2.4 \mathrm{mg} / \mathrm{kg}$ and were evaluable for efficacy, 23 of whom had an OR (7 CRs; 16 PRs). These included 14 of 25 patients with DLBCL (4 CRs; $10 \mathrm{PRs}$ ), 7 of 15 with indolent B-cell lymphoma (3 CRs; 4 PRs) and 2 of 2 with MCL (all PRs). Overall, polatuzumab vedotin $2.4 \mathrm{mg} / \mathrm{kg}$ recipients had a median DoR of 6.2 months and a median PFS of 5.7 months. In this study, which was open-label and conducted in the USA, Canada, France and The Netherlands, polatuzumab vedotin was administered intravenously in 21-day cycles [23].

Data from a similarly-designed phase 1 trial (JO29138) in Japanese patients with relapsed/refractory B-cell NHL supported these findings, with intravenous polatuzumab vedotin (1.0 or $1.8 \mathrm{mg} / \mathrm{kg}$ every 21 days) being associated with an OR rate of 43\% [37]. Data are available from four patients with FL and three with DLBCL [37].

\subsection{Adverse Events}

Intravenous polatuzumab vedotin, as monotherapy or in combination with immunotherapy (with or without chemotherapy or immunomodulation), had an acceptable tolerability profile in patients with NHL in phase 1 or $1 \mathrm{~b} / 2$ trials.

When polatuzumab vedotin $(0.1-2.4 \mathrm{mg} / \mathrm{kg})$ was assessed as monotherapy in patients with NHL in a phase 1 study (NCT01290549), treatment-emergent AEs (TEAEs) were generally grade $1-2$ in severity [23]. Among the 45 patients who received polatuzumab vedotin at $2.4 \mathrm{mg} / \mathrm{kg}$ (the dose recommended for phase 2 assessment), the grade 3/4 TEAEs that occurred most frequently (i.e. in more than two recipients) were neutropenia ( $40 \%$ of patients), anaemia (11\%) and peripheral sensory neuropathy (9\%). Peripheral sensory neuropathy was the AE that most often resulted in polatuzumab vedotin being discontinued (24\% of patients) and neutropenia was the most common reason for its dosing being delayed (24\%). Serious TEAEs occurred in 38\% of patients (17 of 45), the most common of which were diarrhoea, lung infection, lung disorder and disease progression (two patients each) [23]. Polatuzumab vedotin monotherapy $(1.0$ or $1.8 \mathrm{mg} / \mathrm{kg})$ displayed a generally similar tolerability profile in Japanese patients with B-cell NHL in another phase 1 trial (JO29138); no dose-limiting toxicities were observed [37].

AEs were of a generally similar nature with polatuzumab vedotin-containing immunotherapy regimens, with or without concomitant chemotherapy or immunomodulation, in patients with NHL [23-26, 29, 31, 36].

For instance, among patients with relapsed/refractory DLBCL in the phase $1 \mathrm{~b} / 2$ GO29365 study, the adverse reactions that occurred most frequently ( $>20 \%$ incidence) with polatuzumab vedotin $(1.8 \mathrm{mg} / \mathrm{kg})$, bendamustine plus rituximab therapy and with an incidence $\geq 5 \%$ greater than with bendamustine plus rituximab were neutropenia ( $49 \%$ vs. $44 \%$ ), thrombocytopenia ( $49 \%$ vs. $33 \%$ ), anaemia ( $47 \%$ vs. $28 \%$ ), peripheral neuropathy ( $40 \%$ vs. $8 \%$ ), diarrhoea ( $38 \%$ vs. $28 \%$ ), pyrexia ( $33 \%$ vs. $23 \%$ ), decreased appetite ( $27 \%$ vs. $21 \%$ ) and pneumonia ( $22 \%$ vs. $15 \%$ ) [3]. The most common grade $\geq 3$ adverse reactions with the polatuzumab vedotin-containing regimen likewise included neutropenia ( $42 \%$ vs. $36 \%$ with bendamustine plus rituximab), thrombocytopenia ( $40 \%$ vs. $26 \%$ ), anaemia ( $24 \%$ vs. $18 \%$ ) and pneumonia ( $16 \%$ vs. $2.6 \%$ ), the latter of which was fatal in two recipients (vs. one bendamustine plus rituximab recipient). Select laboratory abnormalities that occurred most commonly with the polatuzumab vedotin-containing regimen and with $\geq 5 \%$ greater incidence than with bendamustine plus rituximab included increased creatinine ( $87 \%$ vs. $77 \%$ ) and decreased neutrophil count (78\% vs. $56 \%$ ), haemoglobin (78\% vs. $62 \%$ ) and platelet count (76\% vs. $64 \%$ ) among all grade abnormalities, and decreased lymphocyte count (87\% vs. $82 \%)$, neutrophil count ( $61 \%$ vs. $33 \%$ ) and platelet count (31\% vs. $26 \%$ ) among grade $3 / 4$ abnormalities [3]. In this analysis, $64 \%$ of polatuzumab vedotin, bendamustine plus rituximab recipients experienced serious adverse reactions [the most common of which were pneumonia (16\%), febrile neutropenia (11\%), pyrexia (9\%) and sepsis (7\%)] and 7\% died from adverse reactions within 90 days of receiving their last treatment [3]. Similar tolerability findings were reported with polatuzumab vedotin, bendamustine plus rituximab in an analysis of GO29365 that included both DLBCL and FL patients [29], and with polatuzumab vedotin, bendamustine plus either rituximab or obinutuzumab in other analyses of the trial $[3,26]$. 
With regard to its use in other immunochemotherapy regimens, polatuzumab vedotin in combination with rituximab or obinutuzumab plus CHP in patients with NHL in a phase 1b/2 trial (NCT01992653) [31] displayed a tolerability profile similar to that established for rituximab or obinutuzumab plus CHOP (i.e. CHP plus vincristine). For example, the most common grade $\geq 3$ TEAEs across all polatuzumab vedotin doses assessed in these regimens included neutropenia (32\% of 82 evaluable patients), febrile neutropenia $(17 \%)$, thrombocytopenia (10\%), leukocytosis (9\%), leukopenia (6\%), anaemia (5\%) and pneumonia (5\%) [31]. Some of these TEAEs were also among those that occurred most frequently at grade $\geq 3$ severity when polatuzumab vedotin (1.4 or $1.8 \mathrm{mg} / \mathrm{kg}$ ) was used in combination with obinutuzumab plus lenalidomide in patients with FL in another phase $1 \mathrm{~b} / 2$ trial (NCT02600897): neutropenia (46\%), thrombocytopenia (17\%), anaemia (12\%) and infections (12\%) [36].

Guidelines for the management of peripheral neuropathy, infusion-related reactions and myelosuppression are provided in the manufacturer's prescribing information, as are recommendations pertaining to the use of prophylactic medications (e.g. granulocyte colony-stimulating factor for neutropenia) [3].

\subsection{Ongoing Clinical Trials}

A phase 3 trial in patients with previously untreated DLBCL comparing polatuzumab vedotin plus rituximab-CHP with rituximab-CHOP (NCT03274492; POLARIX) was initiated in November 2017 by Hoffmann-La Roche; it is expected to enroll 875 participants and be completed in 2025. In addition, phase $1 \mathrm{~b} / 2$ trials are evaluating polatuzumab vedotin in combination with other immunochemotherapy regimens in patients with previously untreated DLBCL (mosunetuzumab-CHP or rituximab-CHP; NCT03677141) or in patients with relapsed/refractory DLBCL or FL [bendamustine plus either rituximab (NCT02257567; JapicCTI184048) or obinutuzumab (NCT02257567); venetoclax plus either rituximab or obinutuzumab (NCT02611323)].

Polatuzumab vedotin regimens that are free from standard chemotherapy agents are also being evaluated in phase $1 \mathrm{~b} / 2$ trials. One of the studies is evaluating polatuzumab vedotin in combination with the immunomodulating agent lenalidomide plus either rituximab or obinutuzumab as induction therapy in patients with relapsed/refractory DLBCL or FL (NCT02600897), while the other study is comparing mosunetuzumab (alone or in combination with polatuzumab vedotin) with polatuzumab vedotin, bendamustine plus rituximab in patients with relapsed/refractory DLBCL and with investigator-chosen therapy in patients with relapsed/ refractory FL (NCT03671018).

\section{Current Status}

Polatuzumab vedotin received its first global approval on 10 June 2019 in the USA for use in combination with bendamustine plus rituximab as a treatment for adults with relapsed/refractory DLBCL, not otherwise specified, after at least two prior therapies [4].

\section{Compliance with Ethical Standards}

Funding The preparation of this review was not supported by any external funding.

Conflict of interest During the peer review process the manufacturer of the agent under review was offered an opportunity to comment on the article. Changes resulting from any comments received were made by the authors on the basis of scientific completeness and accuracy. Emma Deeks is a salaried employee of Adis International Ltd/Springer Nature, is responsible for the article content and declares no relevant conflicts of interest.

Open Access This article is distributed under the terms of the Creative Commons Attribution-NonCommercial 4.0 International License (http://creativecommons.org/licenses/by-nc/4.0/), which permits any noncommercial use, duplication, adaptation, distribution and reproduction in any medium or format, as long as you give appropriate credit original author(s) and the source, provide a link to the Creative Commons licence and indicate if changes were made.

\section{References}

1. ADC Review. What are antibody-drug conjugates (ADCs)? J Antibody Drug Conjugates. 2019. https://www.adcreview.com/ the-review/antibody-drug-conjugates/what-are-antibody-drugconjugates/. Accessed 28 Jun 2019.

2. The Human Protein Atlas. CD79B. 2019. https://www.proteinatlas.org/ ENSG00000007312-CD79B/pathology. Accessed 28 June 2019.

3. Genentech Inc. Polivy ${ }^{\mathrm{TM}}$ (Polatuzumab vedotin-piiq) for injection, for intravenous use: US Prescribing Information. 2019. https:// www.fda.gov. Accessed 28 June 2019.

4. U.S. Food and Drug Administration. FDA approves polatuzumab vedotin-piiq for diffuse large B-cell lymphoma. 2019. https:// www.fda.gov/drugs/resources-information-approved-drugs/fdaapproves-polatuzumab-vedotin-piiq-diffuse-large-b-cell-lymph oma. Accessed 28 Jun 2019.

5. Seattle Genetics Inc. Seattle Genetics announces antibody-drug conjugate agreement with Genentech [media release]. $22 \mathrm{Apr}$ 2002. http://www.seattlegenetics.com.

6. Seattle Genetics, Inc. Seattle Genetics establishes preferred antibody-drug conjugate manufacturing relationship with Albany Molecular Research, Inc [media release]. 13 Jul 2004. http://www. seattlegenetics.com/.

7. Seattle Genetics. Seattle Genetics receives additional $\$ 10$ million from Genentech under antibody-drug conjugate collaboration [media release]. 19 Dec 2003. http://www.seattlegenetics.com.

8. Seattle Genetics, Inc. Seattle Genetics and Genentech expand antibody-drug conjugate collaboration [media release]. 9 Nov 2004. http://www.seattlegenetics.com.

9. Seattle Genetics, Inc. Seattle Genetics announces extension of antibody-drug conjugate collaboration with Genentech [media release]. 28 Mar 2007. http://www.seattlegenetics.com. 
10. Seattle Genetics, Inc. Seattle Genetics to receive $\$ 9.5$ million payment from Genentech to extend antibody-drug conjugate collaboration [media release]. $20 \mathrm{Apr} 2010$. http://www.seattlegen etics.com.

11. Seattle Genetics, Inc. Seattle Genetics expands antibody-drug conjugate collaboration with Genentech [media release]. 3 Aug 2010. http://www.seattlegenetics.com.

12. Pfeifer M, Zheng B, Erdmann T, et al. Anti-CD22 and anti-CD79B antibody drug conjugates are active in different molecular diffuse large B-cell lymphoma subtypes. Leukemia. 2015;29(7):1578-86.

13. Tiwari AA, Ayello J, Van De Ven C, et al. Antibody drug conjugates (anti-CD79b-vc-MMAE, polatuzumab vedotin) exhibit enhanced cell death targeted to CD79b + Burkitt lymphoma (BL) and primary mediastinal large B-cell lymphoma (PMBL) [abstract no. 579]. Cancer Res. 2016;76(14 Suppl). https://doi. org/10.1158/1538-7445.AM2016-579.

14. Tiwari AA, Edani D, Azmy C, et al. Enhanced in vitro/in vivo cytotoxicity against Burkitt lymphoma/primary mediastinal large B cell lymphoma by polatuzumab vedotin (hu-anti-CD79bvc-MMAE, PV) alone or in combination with obinutuzumab [abstract no. 1788]. Cancer Res. 2018;78(13 Suppl). https://doi. org/10.1158/1538-7445.AM2018-1788.

15. Amin DN, Oeh J, Zindal A, et al. Anti-tubulin antibody drug conjugates potentiate venetoclax activity in non-Hodgkin lymphoma by targeting MCL-1 [abstract no. 880]. Cancer Res. 2018;78(13 Suppl). https://doi.org/10.1158/1538-7445.AM2018-880.

16. Tiwari A, Edani D, Ayello J, et al. Polatuzumab vedotin alone or in combination with obinutuzumab synergistically enhances in vitro cytotoxicity and cytokine release against CD20 +/CD79B + burkitt lymphoma (BL)/primary mediastinal large B cell lymphoma (pmbl) [abstract]. Blood. 2017;130(Suppl 1):1540.

17. Lu D, Girish S, Marchand M, et al. Concentration-QTc analysis of polatuzumab vedotin in patients with B-cell hematologic malignancies [abstract no. PC-02]. Clin Pharmacol Ther. 2016;99(S1):S5.

18. Lu D, Jin JY, Gibiansky L, et al. Exposure-response analysis to assist selection of dose and treatment duration for polatuzumab vedotin as a single agent or in combination with rituximab for the treatment of B-cell lymphoma [abstract]. Blood. 2015;126(23):1525.

19. Shi R, Lu T, Ding H, et al. Ethnicity sensitivity assessment of polatuzumab vedotin pharmacokinetics in relapsed-refractory non-hodgkin lymphoma [abstract no. PII-102]. Clin Pharmacol Ther. 2019;105(S1):S71.

20. Lu D, Jin JY, Gibiansky L, et al. Population pharmacokinetics and exposure-response assessment of anti-CD79B antibody drug conjugate in patients: interim analysis results [abstract no. PII095]. Clin Pharmacol Ther. 2015;97(S1):S88-9.

21. Shemesh CS, Agarwal P, Lee C, et al. Pharmacokinetics and drug interaction potential of polatuzumab vedotin in combination with R/G-CHP in patients with B-cell non-hodgkin lymphoma [abstract no. PII-101]. Clin Pharmacol Ther. 2019;105(S1):S71

22. Agarwal P, Lu D, Samineni D, et al. Clinical pharmacokinetics (PK) of polatuzumab vedotin (Pola) in combination with rituximab (R), obinutuzumab $(\mathrm{G})$, cyclophosphamide $(\mathrm{C})$, doxorubicin $(\mathrm{H})$ and bendamustine (B) in $1 \mathrm{~L}$ or relapsed/refractory (R/R) non-Hodgkin's lymphoma (NHL) patients [abstract no. 283]. Pharmacother. 2017;37(12):e203.

23. Palanca-Wessels MC, Czuczman M, Salles G, et al. Safety and activity of the anti-CD79B antibody-drug conjugate polatuzumab vedotin in relapsed or refractory B-cell non-Hodgkin lymphoma and chronic lymphocytic leukaemia: a phase 1 study. Lancet Oncol. 2015;16(6):704-15.

24. Morschhauser F, Flinn IW, Advani R, et al. Polatuzumab vedotin or pinatuzumab vedotin plus rituximab in patients with relapsed or refractory non-Hodgkin lymphoma: final results from a phase 2 randomised study (ROMULUS). Lancet Haematol. 2019;6(5):e254-65.

25. Phillips T, Brunvand M, Chen A, et al. Polatuzumab vedotin combined with obinutuzumab for patients with relapsed or refractory non-hodgkin lymphoma: preliminary safety and clinical activity of a phase Ib/II study [abstract]. Blood. 2016;128(22):622.

26. Matasar M, Herrera AF, Kamdar M, et al. Polatuzumab vedotin plus bendamustine and rituximab or obinutuzumab in relapsed/ refractory follicular lymphoma or diffuse large B-cell lymphoma: updated results of a phase 1B/2 study [abstract no. S468]. Haematologica. 2017;102(s2):173.

27. Sehn LH, Herrera AF, Matasar MJ, et al. Polatuzumab vedotin (POLA) plus bendamustine (B) with rituximab (R) or obinutuzumab $(\mathrm{G})$ in relapsed/refractory $(\mathrm{R} / \mathrm{R})$ diffuse large B-cell lymphoma (DLBCL): updated results of a phase (PH) IB/II study [abstract]. Blood. 2018;132(Suppl 1):1683.

28. McMillan A, Sehn L, Herrera A, et al. Updated results from a phase Ib/II study in patients (pts) with relapsed/refractory diffuse large B-cell lymphoma (R/R DLBCL) treated with polatuzumab vedotin (Pola) plus bendamustine (B) with rituximab (R) or obinutuzumab (G) [abstract no. BSH19-PO-079]. Br J Haematol. 2019;185(S1):80.

29. Sehn LH, Kamdar M, Herrera AF, et al. Randomized phase 2 trial of polatuzumab vedotin (Pola) with bendamustine and rituximab (BR) in relapsed/refractory (r/r) FL and DLBCL [abstract no. 7507]. J Clin Oncol. 2018;36(15 Suppl).

30. Sehn LH, Kamdar M, Herrera AF, et al. Adding polatuzumab vedotin (POLA) to bendamustine and rituximab (BR) treatment improves survival in patients with relapsed/refractory dlbcl: results of a phase 2 clinical trial [abstract no. S802]. HemaSphere. 2018;2(S1):348-9.

31. Tilly H, Morschhauser F, Bartlett NL, et al. Polatuzumab vedotin in combination with immunochemotherapy in patients with previously untreated diffuse large B-cell lymphoma: an open-label, non-randomised, phase 1b-2 study. Lancet Oncol. 2019. https:// doi.org/10.1016/s1470-2045(19)30091-9.

32. Forero-Torres A, Kolibaba KS, Tilly H, et al. Polatuzumab vedotin combined with obinutuzumab, cyclophosphamide, doxorubicin, and prednisone (G-CHP) for patients with previously untreated diffuse large b-cell lymphoma (DLBCL): updated results of a phase IB/II study [abstract]. Blood. 2017;130(Suppl 1):4120.

33. Bartlett NL, Chen AI, Kolibaba KS, et al. Polatuzumab vedotin combined with rituximab, cyclophosphamide, doxorubicin, and prednisone (R-CHP) for patients with previously untreated diffuse large b-cell lymphoma (DLBCL): preliminary results of a phase Ib dose-escalation. Blood. 2015;126(23): 2726.

34. Tilly H, Sharman J, Bartlett N, et al. Pola-R-CHP: polatuzumab vedotin combined with rituximab, cyclophosphamide, doxorubicin, prednisone for patients with previously untreated diffuse large B-cell lymphoma [abstract no. S106]. Haematologica. 2017;102(s2):4.

35. Tilly H, Morschhauser F, Bartlett NL, et al. Polatuzumab vedotin combined with rituximab, cyclophosphamide, doxorubicin, and prednisone (R-CHP) for patients with previously untreated diffuse large B-cell lymphoma (DLBCL): updated results of a phase Ib/II study [abstract]. Blood. 2016;128(22):1853.

36. Diefenbach C, Kahl B, Banerjee L, et al. Polatuzumab vedotin (Pola) + obinutuzumab $(\mathrm{G})$ and lenalidomide (Len) in patients (pts) with relapsed/refractory (R/R) follicular lymphoma (FL): interim analysis of a phase $\mathrm{Ib} / \mathrm{II}$ trial [abstract no. 7505]. J Clin Oncol. 2019;37(15 Suppl).

37. Hatake K, Kinoshita T, Terui Y, et al. A phase I pharmacokinetic and safety study of polatuzumab vedotin in Japanese patients with relapsed/refractory B-cell non-Hodgkin lymphoma: a comparison with non-Japanese DCS4968 g study [abstract no. e19070]. J Clin Oncol. 2016;34(15 Suppl). 